\title{
Advances and Challenges in Oral Biofilm Control
}

\author{
Beatriz Helena Dias Panariello ${ }^{1}$ Cecilia Atem Gonçalves de Araújo Costa ${ }^{2}$. \\ Ana Cláudia Pavarina ${ }^{1} \cdot$ Sérgio Lima Santiago ${ }^{2} \cdot$ Simone Duarte $^{1}$
}

Published online: 14 January 2017

(C) Springer International Publishing AG 2017

\begin{abstract}
Purpose of Review The paper aims to critically study the literature published over the past 3 years as relevant to oral biofilm control. The emphasis of this review is the interests and importance of new findings, seeking the following answers: (i) what is the major challenge in oral biofilm control?, (ii) what are the new anti-biofilm approaches?, and (iii) what are the further researches?

Recent Findings In addition to mechanical plaque removal and the use of chemical agents against pathogenic biofilm, there is a need for development of new anti-biofilm approaches. The majority of the new studies aiming to control oral biofilm have been performed with the characterization of the extracellular matrix components. Exopolysaccharides (EPS), proteins, lipids, nucleic acids (eDNA), lipoteichoic acids (LTA), and lipopolysaccharides have been identified in the matrices of bacterial biofilms and are considered the current targets to oral biofilm control.

Summary The extracellular matrix is essential for the existence of the biofilm and by its virulence both in bacterial and fungal pathogens. The better understanding of the biomechanical properties of the EPS matrix is the main advance and is leading to new chemical and/or biological approaches to remove or disorganize cariogenic biofilms. Recently,
\end{abstract}

This article is part of the Topical Collection on Microbiology

Simone Duarte

sd84@nyu.edu; sduarte@nyu.edu

1 Department of Dental Materials and Prosthodontics, São Paulo State University (UNESP), Araraquara Dental School, Rua Humaitá, 1680, 14801-903, Araraquara, São Paulo, Brazil

2 Department of Restorative Dentistry, Faculty of Pharmacy, Dentistry and Nursing, Federal University of Ceará, Rua Monsenhor Furtado, s/n. Rodolfo Teófilo 60430-355 Fortaleza, Ceará, Brazil researches are focusing on the extracellular matrix for oral biofilm control with further clinical applicability.

Keywords Oral biofilms $\cdot$ Oral microbiota $\cdot$ Extracellular matrix $\cdot$ Biofilm control $\cdot$ Dental caries $\cdot$ Oral fungi

\section{Introduction}

The oral cavity has several distinct habitats that provide different ecological conditions for colonization and growth of microorganisms, such as the mucosal surfaces and teeth. The entirety of microorganisms found in the oral cavity is referred as the oral microbiota, or oral microbiome, defined as "all microorganisms that are found on or in the human oral cavity and its contiguous extensions" [1]. The mouth facilitates the growth of a characteristic resident microbiota that possesses archae, viruses, bacteria, and fungi [2]. Temperature, $\mathrm{pH}$, atmosphere, the host defenses and host genetics may influence the composition of the oral microbiota [3]. There is a symbiotic relationship between the resident oral microbiota and the healthy host that is regulated by active host-microbe cross talk [3]. Some changes in oral environmental conditions may disrupt the symbiotic relationship between the host and its resident microbes, increasing the risk of disease [3]. Microorganisms in oral microbiota can be organized as a biofilm. The oral biofilm is the basis of many oral diseases; including dental caries, periodontal disease and endodontic infections [4]. Besides, there is evidence suggesting that oral microorganisms may be related to the pathogenesis of systemic disease including cardiovascular disease, rheumatoid arthritis, respiratory disease, and other conditions [4].

Regarding bacteria, the genera Streptococcus, Lactobacillus, Actinomycetes, Propionibacterium, and Veillonella are highly abundant in the oral microbiome of 
plaque from caries-active adults [5]. When there is an unbalance in the immune system of the host, the oral microbiota is modified and fungi may invade the oral tissues causing infections, such as candidiasis. More than 100 species of fungi were identified in samples from the oral cavity [2], including Candida spp., Aspergillus spp., Fusarium spp., Cryptococcus spp., and Malassezia spp. [6, 7].

It is noted that although fungi have significant roles in the development of biofilm-related diseases, most of the studies on the oral microbiota are limited to bacteria. However, the growing importance of fungi species, mainly Candida spp. as an opportunist pathogen, especially in immunocompromised individuals, has been reported [8].

\section{Extracellular Matrix: a Challenge in Biofilm Control}

Biofilm growth begins when planktonic microbial cells adhere to a surface and start secretion of extracellular matrix components. Exopolysaccharides (EPS), proteins, lipids, nucleic acids, lipoteichoic acids (LTA), and lipopolysaccharides have been identified in the matrices of bacterial biofilms [9]. Infections caused by Candida spp. are also associated with biofilm formation and with the development of an extracellular matrix (ECM) made up of secreted microbial and hostderived substances as well as cell lysis products [10]. Proteins, lipids, carbohydrates, and nucleic acids were identified in these matrices too, in the proportion of $55 \%$ protein, $25 \%$ carbohydrate, $15 \%$ lipid, and $5 \%$ nucleic acid for Candida albicans strain K1 [11••].

In the oral microbiome, besides Streptococcus mutans, many organisms are equally acidogenic and aciduric; however, $S$. mutans is the prime EPS producer, which is important for the establishment of virulent biofilms. Furthermore, S. mutans quickly modulate the formation of cariogenic biofilms when dietary sucrose and starch are present [12••].

In bacterial biofilms, the matrix polysaccharides include mainly glucose and fructose homopolymers that are synthesized from dietary sucrose by glucosyltransferases (Gtfs) and fructosyltransferases. These enzymes are secreted by Streptococcus spp., Lactobacillus spp., and Actinomyces spp. [13]. Glucans synthesized by Gtfs can act as binding sites for $S$. mutans and other organisms. Currently, there are three genetically different enzymes that are produced by bacteria; they are GtfB, GtfC, and GtfD. Each one synthesizes a structurally distinct EPS using sucrose as substrate [14]. The polymers formed on surfaces provide additional non-mammalian bacterial binding sites in the pellicle to increase accumulation of microorganisms, mainly S. mutans [15]. Gtfs, especially $\mathrm{GtfB}$, also bind other oral microorganisms, like Actinomyces viscosus, Lactobacillus casei, and C. albicans, converting them into glucan producers $[15,16]$. GtfC is found in the pellicle in an active form [17]. GtfD produces soluble glucans that act as primers for $\mathrm{GtfB}$ and as a reserve source of energy [17]. The knowledge of how the reactions of Gtfs affect the production and structure of EPS in situ, as well as the supply of microbial binding sites and subsequent matrix assembly will increase the understanding about the mechanisms of cariogenic biofilm installation [18].

Beyond polysaccharides, $S$. mutans also releases extracellular DNA (eDNA) and lipoteichoic acid (LTA), which can contribute with matrix development $[12 \bullet \bullet, 17]$. eDNA is an important extracellular matrix (ECM) component of fungal and bacterial biofilms $[19,20]$. In bacteria, this component enhances EPS synthesis locally, increasing the adhesion of S. mutans to saliva-coated apatitic surfaces and the setting of highly cohesive biofilms [12••]. eDNA and other extracellular substances, acting together with EPS, may interfere with the functional properties of the matrix and the virulence of cariogenic biofilms $[12 \bullet \bullet]$. Bacterial biofilm studies suggested that eDNA has a multifactorial purpose, such as nutrient source [21], facilitator of genetic information exchange [22], contributor to biofilm initiation $[22,23]$, to biofilm stability and dispersal [21, 23, 24], and to antimicrobial resistance factor [22], since the negative charge of eDNA sequester cationic antibiotics [25]. Recent research found that eDNA may contribute to the assembly of the matrix by enhancing glucan synthesis and promoting bacterial binding to surfaces $[12 \bullet \bullet, 17,26]$, but the molecular mechanisms that triggers the release and secretion of eDNA and how it is incorporated into the biofilm matrix remain unknown $[12 \bullet \cdot, 17]$.

Environmental changes in the mouth of the host, such as frequent consumption of sucrose, influence biofilm development by providing a substrate for EPS production on the pellicle surface [18]. If exposure to sucrose persists, EPS will be continuously produced forming the body of the matrix and maturation of the biofilm will occur. The EPS formed on surfaces and the development of an extracellular matrix may be related to mechanical properties of biofilms, including adhesive strength and cohesiveness $[12 \bullet, 17]$. However, it is yet to be determined when and how EPS modulate adhesive and cohesive forces of the matrix, which are fundamental properties for the mechanical stability of biofilms $[12 \bullet \cdot, 17]$. There is a need to better understand the biomechanical properties of the EPS matrix to improve chemical and/or biological approaches to remove and/or disorganize cariogenic biofilms [17].

Bacteria have mechanisms to recognize receptors on host cell surfaces or on components of the EPS [27]. Bacterial species do not act alone; attachment by primary colonizers provides new receptors which may be recognized by secondary colonizers leading to co-aggregation, that is, the adhesion of different bacterial species to one another [27]. If nutrient sources are available, biofilms can grow into larger structures than individual cells. On the other hand, if resources become depleted, 
bacteria can return to the planktonic phase and disperse to encounter new locations with superior resource availability [28].

Cell-to-cell binding between microorganisms plays a crucial role in integrating secondary colonizers into oral biofilms and building complex networks of interacting microbial cells [27]. Microorganisms entering the mouth have to attach to a surface to evade clearance by salivary flow from the mouth to the digestive tract [27]. Adherence of pathogenic microorganisms to host tissues initiates the disease process [19]. The attached bacteria synthesize a variety of extracellular polymers to structure the biofilm matrix, that retain and bind many molecules, including enzymes, and so the matrix is biologically active [19].

The matrix is considered essential for the existence of the biofilm behavior and full expression of virulence by bacterial pathogens [12••]. Matrix constituents could affect the diffusion of substances in and out of the biofilm, helping to create a diverse range of microenvironments within the biofilm [18]. Concerning biofilm settlement, the resident microorganisms enmeshed and protected in the matrix are resistant to antimicrobials and are highly acidogenic/acid-tolerant, making them difficult to remove, becoming reservoirs for pathogens and toxins [17]. Thereby, the matrix can be considered the biggest challenge on the oral biofilm control.

\section{Advances in Oral Biofilm Control}

In addition to mechanical plaque removal and the use of chemical agents against biofilm pathogens, the development of new anti-biofilm approaches is necessary. Polysaccharides and eDNA, which are crucial structural and functional constituents of the biofilm matrix, seem to be a good target for future therapeutic strategies [12••, 13, 17, 18, 21, 23, 25, 26, 28-30]. The control of the biofilm that does not affect the viability of commensal oral bacteria, like the use of enzymes, represents a promising approach for therapy of oral diseases [13]. The possibilities of using enzymes should be determined, including the use of matrix-targeting enzymes to help biofilm penetration by antimicrobial agents for which the matrix is a diffusion barrier [29].

As cited before, the eDNA is an important ECM component of both fungal and bacterial biofilms and it is related to antimicrobial resistance. So, targeting eDNA via DNAse might be a strategy to improve the penetration of drugs into the extracellular matrix of these microorganisms. In the oral cavity, strategies to deliver DNase enzymes would need to ensure that the enzymes are safe for use in humans [30]. Besides that, it is important to ensure that the enzyme will reach the oral biofilm effectively, that it is active when in contact with the oral biofilm, that its activity is retained for long enough to disturb the biofilm, and that the enzyme is stable for a reasonable period
[30]. It is necessary to understand how eDNA interacts within the biofilm matrix. Many research groups are addressing this important question, thus some eDNAbinding compounds, polysaccharides, and proteins are emerging in the literature [25]. Recently, ciprofloxacinloaded poly (lactic-co-glycolic acid) nanoparticles functionalized with DNase I were fabricated and their antibiofilm activity was assessed against Pseudomonas aeruginosa biofilms. DNase I-activated nanoparticles successfully reduced established biofilm mass, size, and living cell density, as observed in a dynamic environment in a flow cell biofilm assay [31•].

Another novel strategy to control plaque-biofilms was described by using atmospheric-pressure cold plasma (ACP), since it has the ability to disrupt the biofilm matrix and rupture cell structure. The cold plasma is generated by gas ionization at atmospheric pressure and low temperature; producing reactive oxygen species, reactive nitrogen species, UV radiation, ions, electrons, excited molecules, and electromagnetic field. Antimicrobial effect of ACP was shown on single- and dualspecies biofilms of C. albicans and Staphylococcus aureus as well as biological safety of ACP on in vitro reconstituted oral epithelium [32]. Another recent technique that showed relevant results inhibiting matrix-rich biofilm development was the blue light treatment without a photosensitizer in $S$. mutans biofilms. When the biofilms were treated with twice-daily blue light, EPS insoluble was reduced and the reduction was even more effective than the twice-daily application of the "gold-standard" $0.12 \%$ chlorhexidine [33].

\section{Conclusions}

The challenge of developing effective therapies to control oral biofilms is that topically introduced agents have to evade the fast clearance from biofilm-tooth interfaces while targeting biofilm microenvironments. Furthermore, EPS matrix and acidification of biofilm microenvironments are associated to cariogenic biofilm virulence. The improvement of the bioavailability and retention of antibacterial agents at the dental surfaces and within the biofilm is needed. Therefore, the acquaintance of biofilm matrix assembly and changes in the biofilm microenvironment allows the discovering of new approaches against infectious biofilms [34].

Acknowledgements This work was supported by a São Paulo Research Foundation (FAPESP) scholarship 2016/00256-3 to B.H.D.P. and by the Coordination for the Improvement of Higher Education Personnel (CAPES) scholarship 6483-15-1 to C.A.G.A.C.

\section{Compliance with Ethical Standards}

Conflict of Interest All authors declare that they have no conflict of interest. 
Human and Animal Rights and Informed Consent This article does not contain any studies with human or animal subjects performed by any of the authors.

\section{References}

Papers of particular interest, published recently, have been highlighted as:

- Of Importance

•- Of major importance

1. Dewhirst FE, Chen T, Izard J, Paster BJ, Tanner AC, Yu WH, Lakshmanan A, Wade WG. The human oral microbiome. J Bacteriol. 2010;192(19):5002-17. doi:10.1128/JB.00542-10.

2. Willems HM, Xu Z, Peters BM. Polymicrobial biofilm studies: from basic science to biofilm control. Curr Oral Health Rep. 2016;3(1):36-44. doi:10.1007/s40496-016-0078-y.

3. Marsh PD, Devine DA. How is the development of dental biofilms influenced by the host? J Clin Periodontol. 2011;38(Suppl 11):28 35. doi:10.1111/j.1600-051X.2010.01673.x.

4. Maddi A, Scannapieco FA. Oral biofilms, oral and periodontal infections, and systemic disease. Am J Dent. 2013;26(5):249-54.

5. Belda-Ferre P, Alcaraz LD, Cabrera-Rubio R, Romero H, SimónSoro A, Pignatelli M, Mira A. The oral metagenome in health and disease. ISME J. 2012;6(1):46-56. doi:10.1038/ismej.2011.85.

6. Dupuy A, David MS, Li L, Heider TN, Peterson JD, Montano EA, Dongari-Bagtzoglou A, Diaz PI, Strausbaugh LD. Redefining the human oral mycobiome with improved practices in amplicon-based taxonomy: discovery of Malassezia as a prominent commensal. PLoS One. 2014;9(3):e90899. doi:10.1371/journal.pone.0090899.

7. Ghannoum MA, Jurevic RJ, Mukherjee PK, Cui F, Sikaroodi M, Naqvi A, Gillevet PM. Characterization of the oral fungal microbiome (mycobiome) in healthy individuals. PLoS Pathog. 2010;6(1):e1000713. doi:10.1371/journal.ppat.1000713.

8. Tsai HF, Sammons LR, Zhang X, Suffis SD, Su Q, Myers TG, Marr KA, Bennett JE. Microarray and molecular analyses of the azole resistance mechanism in Candida glabrata oropharyngeal isolates. Antimicrob Agents Chemother. 2010;54(8):3308-17. doi:10.1128 /AAC.00535-10.

9. Karatan E, Watnick P. Signals, regulatory networks, and materials that build and break bacterial biofilms. Microbiol Mol Biol Rev. 2009;73(2):310-47. doi:10.1128/MMBR.00041-08.

10. Nobile CJ, Mitchell AP. Microbial biofilms: e pluribus unum. Curr Biol. 2007;17(10):R349-53. doi:10.1016/j.cub.2007.02.035.

11.• Zarnowski R, Westler WM, Lacmbouh GA, Marita JM, Bothe JR, Bernhardt J, Lounes-Hadj Sahraoui A, Fontaine J, Sanchez H, Hatfield RD, Ntambi JM, Nett JE, Mitchell AP, Andes DR. Novel entries in a fungal biofilm matrix encyclopedia. MBio. 2014;5(4):e01333-14. doi:10.1128/mBio.01333-14. This report is the first to decipher the complex and unique macromolecular composition of the Candida biofilm matrix. It demonstrated the clinical relevance of matrix components, and it was showed that multiple matrix components are needed for protection from antifungal drugs

12.• Klein MI, Hwang G, Santos PH, Campanella OH, Koo H. Streptococcus mutans-derived extracellular matrix in cariogenic oral biofilms. Front Cell Infect Microbiol. 2015;5:10. doi:10.3389/fcimb.2015.00010. This manuscript shows that eDNA enhances EPS synthesis locally, increasing the adhesion of S. mutans to saliva-coated apatitic surfaces and the assembly of highly cohesive biofilms

13. Pleszczyńska M, Wiater A, Bachanek T, Szczodrak J. Enzymes in therapy of biofilm-related oral diseases. Biotechnol Appl Biochem. 2016; doi:10.1002/bab.1490.

14. Bowen WH, Koo H. Biology of Streptococcus mutans-derived glucosyltransferases: role in extracellular matrix formation of cariogenic biofilms. Caries Res. 2011;45(1):69-86. doi:10.1159 1000324598

15. Vacca-Smith AM, Bowen WH. Binding properties of streptococcal glucosyltransferases for hydroxyapatite, saliva-coated hydroxyapatite, and bacterial surfaces. Arch Oral Biol. 1998;43(2):103-10. doi:10.1016/S0003-9969(97)00111-8.

16. Gregoire S, Xiao J, Silva BB, Gonzalez I, Agidi PS, Klein MI, Ambatipudi KS, Rosalen PL, Bauserman R, Waugh RE, Koo H. Role of glucosyltransferase B in interactions of Candida albicans with Streptococcus mutans and with an experimental pellicle on hydroxyapatite surfaces. Appl Environ Microbiol. 2011;77(18): 6357-67. doi:10.1128/AEM.05203-11.

17. Koo H, Falsetta ML, Klein MI. The exopolysaccharide matrix: a virulence determinant of cariogenic biofilm. J Dent Res. 2013;92(12):1065-73. doi:10.1177/0022034513504218.

18. Xiao J, Klein MI, Falsetta ML, Lu B, Delahunty CM, Yates 3rd JR, Heydorn A, Koo H. The exopolysaccharide matrix modulates the interaction between 3D architecture and virulence of a mixedspecies oral biofilm. PLoS Pathog. 2012;8(4):e1002623. doi:10.1371/journal.ppat.1002623.

19. Flemming HC, Wingender J. The biofilm matrix. Nat Rev Microbiol. 2010;8(9):623-33. doi:10.1038/nrmicro2415.

20. Martins M, Uppuluri P, Thomas DP, Cleary IA, Henriques M, Lopez-Ribot JL, Oliveira R. Presence of extracellular DNA in the Candida albicans biofilm matrix and its contribution to biofilms. Mycopathologia. 2010;169(5):323-31. doi:10.1007/s11046-0099264-y.

21. Whitchurch CB, Tolker-Nielsen T, Ragas PC, Mattick JS Extracellular DNA required for bacterial biofilm formation. Science. $2002 ; 295(5559): 1487$. doi:10.1126 /science.295.5559.1487.

22. Mulcahy H, Charron-Mazenod L, Lewenza S. Extracellular DNA chelates cations and induces antibiotic resistance in Pseudomonas aeruginosa biofilms. PLoS Pathog. 2008;4(11):e1000213. doi:10.1371/journal.ppat.1000213.

23. Izano EA, Amarante MA, Kher WB, Kaplan JB. Differential roles of poly-N-acetylglucosamine surface polysaccharide and extracellular DNA in Staphylococcus aureus and Staphylococcus epidermidis biofilms. Appl Environ Microbiol. 2008;74(2):470-6. doi:10.1128/AEM.02073-07.

24. Berne C, Kysela DT, Brun YV. A bacterial extracellular DNA inhibits settling of motile progeny cells within a biofilm. Mol Microbiol. 2010;77(4):815-29. doi:10.1111/j.13652958.2010.07267.x.

25. Okshevsky M, Regina VR, Meyer RL. Extracellular DNA as a target for biofilm control. Curr Opin Biotechnol. 2015;33:73-80. doi:10.1016/j.copbio.2014.12.002.

26. Liao S, Klein MI, Heim KP, Fan Y, Bitoun JP, Ahn SJ. Burne RA3, Koo H, Brady LJ, Wen ZT. Streptococcus mutans extracellular DNA is upregulated during growth in biofilms, actively released via membrane vesicles, and influenced by components of the protein secretion machinery. J Bacteriol. 2014;196(13):2355-66. doi:10.1128/JB.01493-14.

27. Nobbs AH, Jenkinson HF, Jakubovics NS. Stick to your gums: mechanisms of oral microbial adherence. J Dent Res. 2011;90(11):1271-8. doi:10.1177/0022034511399096.

28. Nadell CD, Drescher K, Wingreen NS, Bassler BL. Extracellular matrix structure governs invasion resistance in bacterial biofilms. ISME J. 2015;9(8):1700-9. doi:10.1038/ismej.2014.246. 
29. Pleszczyńska M, Wiater A, Janczarek M, Szczodrak J. (1 $\rightarrow 3)-\alpha$ D-Glucan hydrolases in dental biofilm prevention and control: a review. Int J Biol Macromol. 2015;79:761-78. doi:10.1016/j. ijbiomac.2015.05.052.

30. Jakubovics NS, Burgess JG. Extracellular DNA in oral microbial biofilms. Microbes Infect. 2015;17(7):531-7. doi:10.1016/j. micinf.2015.03.015.

31. Baelo A, Levato R, Julián E, Crespo A, Astola J, Gavaldà J, Engel E, Mateos-Timoneda MA, Torrents E. Disassembling bacterial extracellular matrix with DNase-coated nanoparticles to enhance antibiotic delivery in biofilm infections. J Control Release. 2015;209: 150-8. doi:10.1016/j.jconrel.2015.04.028. This study showed that repeated administration of DNase I-coated nanoparticles encapsulating ciprofloxacin was able to target and disassemble
Pseudomonas aeruginosa biofilm by degrading the extracellular DNA that stabilize the biofilm matrix

32. Delben JA, Zago CE, Tyhovych N, Duarte S, Vergani CE. Effect of atmospheric-pressure cold plasma on pathogenic oral biofilms and in vitro reconstituted oral epithelium. PLoS One. 2016;11(5): e0155427. doi:10.1371/journal.pone.0155427.

33. de Sousa DL, Lima RA, Zanin IC, Klein MI, Janal MN, Duarte S. Effect of twice-daily blue light treatment on matrix-rich biofilm development. PLoS One. 2015;10(7):e0131941. doi:10.1371 /journal.pone.0131941.

34. Horev B, Klein MI, Hwang G, Li Y, Kim D, Koo H, Benoit DS. pHactivated nanoparticles for controlled topical delivery of farnesol to disrupt oral biofilm virulence. ACS Nano. 2015;9(3):2390-404. doi:10.1021/nn507170s. 